\title{
Comparison of Orcein and CMA Stained Karyotypes in Three Morphological Forms of Typhonium trilobatum L. (Araceae)
}

\author{
Ashma Ahmed Warasy and Sheikh Shamimul Alam* \\ Department of Botany, University of Dhaka, Dhaka-1000, Bangladesh \\ Received July 15, 2009; accepted October 14, 2009
}

\begin{abstract}
Summary The karyotypes in 3 morphological forms of Typhonium trilobatum (L.) Schott such as the Green form, the Dark purple form and the Light purple form were compared after staining with orcein and CMA. The Green form and the Light purple form were found to possess $2 n=18$ chromosomes. However, $2 n=17$ chromosomes were observed in the Dark purple form. One chromosome in pair IX was absent in this form. The karyotypic features revealed that the Dark purple form might be originated from the Green form as monosomic. The centromeric formula was determined $16 \mathrm{~m}+2 \mathrm{ac}$ in the Green form, $11 \mathrm{~m}+4 \mathrm{sm}+2 \mathrm{ac}$ in the Dark purple form and $13 \mathrm{~m}+5 \mathrm{sm}$ in the Light purple form. The Dark purple form showed more heterogeneous karyotype and may be considered as more advanced than the other 2 forms. No CMA band was found in the Green form. The Dark purple and the Light purple forms were found to possess distinct CMA banding pattern. With the help of CMA banding, it was possible to identify some chromosomes in these 2 forms. Few chromosome pairs in both the forms showed heteromorphicity in respect of CMA banding pattern indicating the occurrence of structural aberration. Morphology, distinct karyotypic features and CMA banding pattern suggested for placing the Green form in different taxonomic rank, while the Dark purple form might be considered as a monosomic variety of Typhonium trilobatum.
\end{abstract}

Key words Fluorescent banding, Karyotype, Typhonium.

The genus Typhonium belongs to the family Araceae consisting of about 37 species (Sriboonma et al. 1994). Members of this genus are widely distributed and well adapted in tropical south, south-east and east Asia. In Bangladesh, these are grown in plain, marshy land and hilly areas of Netrokona, Bandarban, Dinajpur, Sherpur, Gojni, Sylhet, Chittagong districts. The members of this widely distributed genus have some economical and ethno-botanical importance. The tubers and roots are acrid and stimulant at fresh stage. The young leaves, tubers and petioles are used as vegetables, to cure piles, stomach disease, as poultice, to meet the iron deficiency etc. In Bangladesh, 7 taxa of Typhonium have been reported so far (Ara 2001, Ara et al. 2005).

Three specimens of Typhonium with different petiole colour have been collected by Ara et al. (2005) from different places in Bangladesh. The specimen collected from Netrokona has green petiole, the other from Dinajpur with deep purple petiole and that from Bandarban district has light purple petiole. These 3 specimens were named as "Green form", "Dark purple form" and "Light purple form" after their petiole colour. On the basis of their gross morphology, these could be considered as T. trilobatum. However, these forms differed from T. trilobatum in certain features, such as i) the Green form has $17 \mathrm{~cm}$ long spathe where the upper spathe is green in outside and light purple in inside and the lower spathe dark green outside. Their female zone is $1 \mathrm{~cm}$ long and $1 \mathrm{~cm}$ broad with a very shortly stipulate appendix, ii) the Dark purple form has a spathe of $23 \mathrm{~cm}$ long with the deep purple colour outside in upper spathe. The appendix of this form is about $9.2 \mathrm{~cm}$ long and iii) the Light purple form has a $16.5 \mathrm{~cm}$ long spathe where inside the spathe is purple and

\footnotetext{
*Corresponding author, e-mail: ssalam81@yahoo.com
} 
outside green in colour. The female sterile zone of this form is about $0.8 \mathrm{~cm}$ long with a $6.5 \mathrm{~cm}$ long appendix. The above mentioned morphological differences did not allow these 3 specimens to be straightforwardly considered as T. trilobatum.

It is well known that karyotype analysis often plays an important role in determining the taxonomic status of a taxon where the taxonomic parameters are insufficient, because karyotype signifies very stable character and specific for each specimen. However, a problem arises when different taxa possess same chromosome number and almost similar karyotypic features. In this situation, it is hard to distinguish between such taxa by conventional karyotype analysis. Even the consideration of chromosome length, arm ratio, position and number of secondary constrictions are not always sufficient to differentiate individual chromosome. Minute alterations regarding the distribution pattern of GC- and AT-rich repeats in the karyotypes could not be possible to detect through orcein-stained karyotype analysis. Moreover, deletion of heterochromatic regions may change the karyotype of a variety without affecting the morphology (Sumner 1990). In such a case, some other methods may be applied. Staining with DNA base specific fluorochromes is one of such methods. Chromomycin $\mathrm{A}_{3}$ (CMA) is a fluorochrome which binds with GC-rich repetitive sequences of the genome and gives characteristic yellow colour bands. With the help of fluorescent staining it was possible to characterize different karyotypes (Schweizer 1976, Alam and Kondo 1995, Alam et al. 1996, Jessy et al. 2005, Akhter and Alam 2005).

Identification problem was also found in other members of Araceae. For example, Colocasia esculenta has 3 and Xanthosoma violaceum has 2 morphological forms. Taxonomists faced problem to identify these taxa authentically. Latter extensive cytological investigation had been carried out in these taxa. The cytological data indicated sharp difference among the different forms of Colocasia esculenta and Xanthosoma violaceum (Deen and Alam 2002, Alam and Deen 2002).

Recently Huq et al. (2007) tried to solve the taxonomic problem of 3 other forms of $T$. trilobatum (Coarse form, Tall form and Slender form). They reported that the Tall form was quite different from the other 2 forms in respect of their karyotypic features and suggested to place the Tall form in a different taxonomic rank. They also suggested considering the Slender form as a trisomic variety of $T$. trilobatum because of having 1 extra chromosome in their chromosome complement (pair IX).

Therefore, an extensive karyotype analysis should be undertaken to solve the taxonomic problems of these 3 specimens such as the Green form (collected from Netrokona), the Dark purple form (collected from Dinajpur) and the Light purple form (collected from Bandarban). In this investigation, as a continuation of Huq et al. (2007) work, the above 3 specimens were studied cytologically with the following aims:

(i) to compare the karyotypes of 3 forms after staining with orcein and CMA,

(ii) to identify marker chromosomes if any, and

(iii) to ascertain the taxonomic status of 3 forms.

\section{Materials and methods}

Three morphological forms of Typhonium trilobatum were used in this study. These are:

(i) Petiole green in colour.

(ii) Petiole dark purple in colour.

(iii) Petiole light purple in colour.

Healthy roots were collected and pretreated with $0.002 \mathrm{M}$ 8-hydroxyquinoline for $1.10 \mathrm{~h}$ at room temperature $\left(18-20^{\circ} \mathrm{C}\right)$ followed by $15 \mathrm{~min}$ fixation in $45 \%$ acetic acid at $4^{\circ} \mathrm{C}$. These were then hydrolysed in a mixture of $1 \mathrm{~N} \mathrm{HCl}$ and $45 \%$ acetic acid $(2: 1)$ at $60^{\circ} \mathrm{C}$ for $6 \mathrm{~s}$. The root tips were stained and squashed in 1\% aceto orcein. For fluorescent banding, Alam and Kondo's (1995) method was followed with slight modification. After hydrolysing and dissecting, the materials were 
squashed with $45 \%$ acetic acid. The cover glasses were removed quickly on dry ice and allowed to air dry for at least $48 \mathrm{~h}$ before study. The air-dried slides were first pre-incubated in McIlvaine's buffer ( $\mathrm{pH} 7.0)$ for $30 \mathrm{~min}$ followed by Distamycin A $(0.1 \mathrm{mg} / \mathrm{ml})$ treatment for $10 \mathrm{~min}$. The slides were rinsed mildly in Mcllvaine's buffer supplemented with $\mathrm{MgSO}_{4}(5 \mathrm{mM})$ for $15 \mathrm{~min}$. One drop of CMA $(0.1 \mathrm{mg} / \mathrm{ml})$ was added to the materials for $15 \mathrm{~min}$ and rinsed with McIlvaine's buffer with $\mathrm{Mg}^{2+}$ for $10 \mathrm{~min}$. Slides were mounted in $50 \%$ glycerol and kept at $4{ }^{\circ} \mathrm{C}$ for overnight before observation. These were observed under Nikon (UFX-IIA) fluorescent microscope with Blue Violet (BV) filter cassette.

\section{Results and discussion}

\section{Orcein-stained Karyotype}

The Green and Light purple form of T. trilobatum were found to possess $2 n=18$ chromosomes (Figs. 1, 3, 7, 9). Huq et al. (2007) reported the same chromosome number for the Tall form and the Coarse form of T. trilobatum. However, $2 n=17$ chromosomes were found in the Dark purple form (Figs. 2, 8). The karyotype of this form showed that a metacentric chromosome from pair IX was absent (Fig. 11, Table 1). The range of chromosomal length was 1.00-2.65 $\mu \mathrm{m}$ for the Green form and 1.00-2.70 $\mu \mathrm{m}$ for the Dark purple form (Table 1). The range of chromosomal length in these 2 forms was almost same (Table 1). Total length of $2 n$ chromosome complement was $30.60 \mu \mathrm{m}$ in the Green form and $29.10 \mu \mathrm{m}$ in the Dark purple form (Table 1). Thus the total length of $2 n$ chromosome of the Dark purple form was about $1.0 \mu \mathrm{m}$ less than the Green form. The length of the only chromosome in pair IX of the Dark purple form (1 chromosome missed in pair IX) was about $1.00 \mu \mathrm{m}$. This data suggested that the Dark purple form might be originated as a monosomic from the Green form.

Huq et al. (2007) reported a trisomic form of T. trilobatum (Slender form). The extra chromosome was a regular member of pair IX of that form. The most probable reason for this kind of numerical aberration was non disjunction of chromosome in pair IX. It may be suspected that during meiosis members of pair IX did not segregate and thus move to a pole. This gamete fertilized with a normal opposite gamete resulting the development of this slender (trisomic) form.

Huq et al. (2007) finding indicated the presence of a monosomic plant as well. Because due to non-disjunction of pair IX, some gametes did not have a member of pair IX and thus may result a monosomic plant. The Dark purple form was found to possess $2 n=17$ chromosomes. The karyotype showed that pair IX was lacking a chromosome (Fig. 11). This result suggested to consider the specimen as monosomic (as per expectations).

The centromeric formula was $16 \mathrm{~m}+2 \mathrm{ac}$ in the Green form, $11 \mathrm{~m}+4 \mathrm{sm}+2 \mathrm{ac}$ in the Dark purple form and $13 \mathrm{~m}+5 \mathrm{sm}$ in the Light purple form (Table 1). Acrocentric chromosomes were found in the Green form and the Dark purple form whereas absent in the Light purple form. The Dark purple form possessed metacentric, sub metacentric and acrocentric chromosomes, thus representing a heterogeneous karyotype. Huq et al. (2007) did not find any acrocentric chromosome in any form of T. trilobatum (Tall, Coarse and Slender form) studied by them. Regarding the presence of acrocentric chromosomes, the karyotypes of the Green form and the Dark purple form differed from the other forms of T. trilobatum.

The heteromorphicity in respect of centromeric position was found in the Light purple form. One member of pair III was submetacentric chromosome and the other metacentric chromosome (Figs. 9, 12, Table 1). The probable reason for this heteromorphicity was deletion of chromosomal part from the long arm of a submetacentric chromosome resulting the formation of metacentric chromosome. 
Table 1. Comparative orcein and CMA-karyotype analysis in 3 forms of Typhonium trilobatum

\begin{tabular}{|c|c|c|c|c|c|c|}
\hline Forms & $2 n$ & $\begin{array}{l}\text { Total length of } \\
2 n \text { chromosome } \\
\text { complement } \\
(\mu \mathrm{m})\end{array}$ & $\begin{array}{l}\text { Karyotype } \\
\text { formulae }\end{array}$ & $\begin{array}{c}\text { Range of } \\
\text { chromosomal } \\
\text { length } \\
(\mu \mathrm{m})\end{array}$ & $\begin{array}{l}\text { No. of } \\
\text { CMA } \\
\text { positive } \\
\text { bands }\end{array}$ & $\begin{array}{l}\% \text { of } \\
\text { area of } \\
\text { CMA } \\
\text { bands }\end{array}$ \\
\hline Green form & 18 & 30.60 & $16 \mathrm{~m}+2 \mathrm{ac}$ & $1.00-2.65$ & - & - \\
\hline Dark purple form & 17 & 29.10 & $11 \mathrm{~m}+4 \mathrm{sm}+2 \mathrm{ac}$ & $1.00-2.70$ & 8 & 25.43 \\
\hline Light purple form & 18 & 32.10 & $13 m+5 s m$ & $1.00-3.25$ & 14 & 49.84 \\
\hline
\end{tabular}

$\mathrm{m}=$ metacentric chromosome, $\mathrm{sm}=$ submetacentric chromosome, $\mathrm{ac}=$ acrocentric chromosome.

\section{CMA-banding}

No CMA-band (positive or negative) was found in the Green form. Moreover, no NOR was observed in this form. Generally NOR show CMA positive. This result indicates the absence of GC-rich repeats.

The Dark purple form and the Light purple form showed certain similarities in CMA-banding pattern (Figs. 8, 9, 11, 12). Such as: i) no CMA-negative band was found, ii) CMA-bands were thick, bright and prominent, iii) few chromosomes were fluoresced along their entire length and iv) relatively short arm of few chromosomes were fluoresced entirely. Huq et al. (2007) reported almost same kind of CMA-banding pattern in the other 3 forms of T. trilobatum. This pattern of CMA-banding indicated that the karyotypes of different forms of T. trilobatum might be derived from common ancestor.

Apart from the similarities mentioned above the karyotypes of the Dark purple form and the Light purple form differed sharply. The Dark purple form possessed 2 entirely, 5 upper terminals and 1 interstitial banded chromosomes. Nine chromosomes of this form did not show any band (Fig. 11, Table 1). On the other hand, 7 entirely and 7 upper terminal banded chromosomes were found in the Light purple form. Four chromosomes of the latter form did not show any band (Fig. 12, Table 1). The percentage of CMA-banded region was $25.43 \%$ and $49.84 \%$ in the Dark purple form and the Light purple form, respectively (Table 1). In contrast, no CMA-band was found in the Green form (Fig. 10, Table 1). Therefore, each form has its own CMA-banded karyotype.

\section{Marker chromosomes}

The shape and size of orcein stain and CMA-banded chromosome were almost similar, however it looks bigger in CMA banding because of fluorescence. In the Dark purple form, both the members of pair VI fluoresced entirely. No other entirely fluoresced chromosome was found in this form. One member of pair II had a centromeric thick and prominent band (Fig. 11). No other centromeric banded chromosome was found in this form. Therefore, the entirely fluoresced and centromeric banded chromosomes were the marker chromosomes for this form.

In the Light purple form, 1 member of pair I had a big CMA-band occupied it's short arm and a portion of long arm including centromere (Fig. 12, arrow). One member of pair III possessed a CMA-band occupying vertically half of the short arm (Fig. 12, arrow). These 2 chromosomes were unique for this form and thus could be used as marker chromosomes.

\section{Taxonomic status of 3 forms}

The karyotypic features and CMA-banding pattern clearly indicated that the Green form was quite different from the rest 2. In this form, no submetacentric chromosome and CMA-band was found (Table 1). On the other hand submetacentric chromosomes and different types of CMA bands were found in the other 2 forms (Figs. 8, 9, 11, 12, Table 1). Huq et al. (2007) found submetacentric 

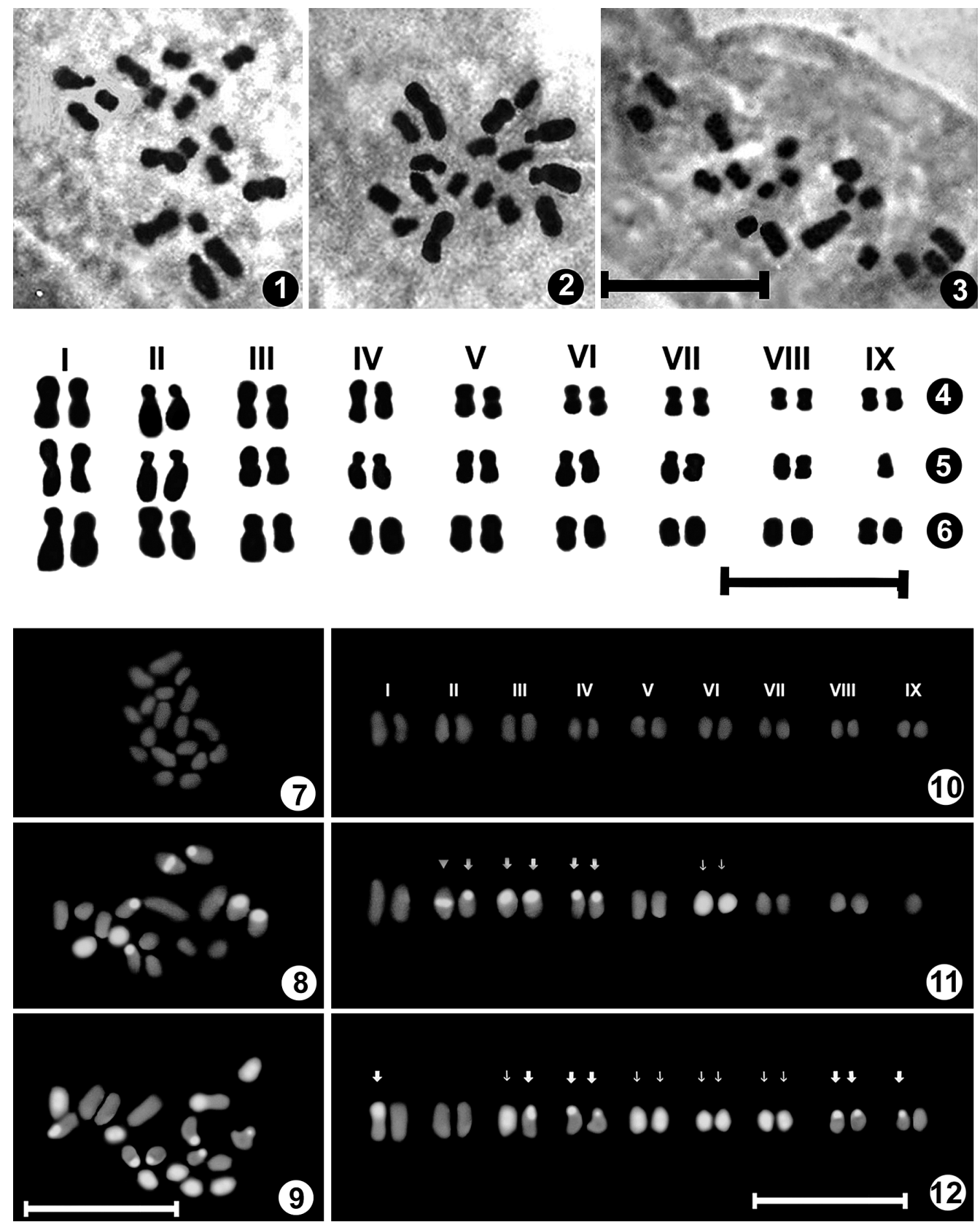

Figs. 1-12. Mitotic metaphase chromosomes and karyotypes of 3 forms in Typhonium trilobatum. 1. Orcein-stained mitotic metaphase of the Green form. 2. Orcein-stained mitotic metaphase of the Dark purple form. 3. Orcein-stained mitotic metaphase of the Light purple form. 4. Orceinstained karyotype of the Green form. 5. Orcein-stained karyotype of the Dark purple form. 6 . Orcein-stained karytype of the Light purple form. 7. CMA-stained mitoric metaphase of the Green form. 8. CMA-banded karyotype of the Dark purple form. 9. CMA-stained mitoric metaphase of the Light purple form.10. CMA-banded karyotype of the Green form. 11. CMAbanded karyotype of the Dark purple form. 12. CMA-banded karyotype of the Light purple form. Bar $=10 \mu \mathrm{m}$. 
chromosomes and CMA-band in the three other forms of $T$. trilobatum. Therefore, the Green form should not be treated as T. trilobatum. Distinct morphology, karyotypic features and CMA-banding pattern suggested for placing this form in different taxonomic rank. The Dark purple form possessed $2 n=17$ chromosomes. From this point of view this form could be considered as monosomic variety of $T$. trilobatum. The morphological feature of Light purple form together with CMA-banding pattern and percentage of GC-rich region may suggest for considering this form as a variety of T. trilobatum.

\section{Acknowledgements}

We are grateful to Dr. M. Akhtaruzzaman, Professor of Botany, University of Dhaka, Bangladesh for constructive criticism and going through the manuscript. Sincere thanks to Mrs. Hosne Are, director in charge, Bangladesh National Herbarium, for providing the plant materials and all necessary information about Typhonium trilobatum.

\section{References}

Akter, S. and Alam, Sk. S. 2005. Differential fluorescent banding pattern in three varieties of Cicer arietinum L. (Fabaceae). Cytologia 70: 441-445.

Alam, Sk. S. and Kondo, K. 1995. Differential staining with Orcein, Giemsa, CMA and DAPI for comparative chromosome study of 12 species of Australian Drosera (Droseraceae). Amer. J. Bot. 82: 1278-1286.

—, Kondo, K. and Hoshi, Y. 1996. Study on diffused centromeric nature of Drosera chromosomes. Cytologia 60: $43-47$.

— and Deen, S. S. 2002. Karyotype and isozyme analysis in three forms of Colocasia esculenta (Araceae). Bangladesh J. Bot. 31: 95-98.

Ara, H. 2001. An annotated checklist of Aroids in Bangladesh. Bangladesh J. Plant Taxon. 8: 19-34.

—, Partha, P. and Hassan, A. 2005. New records of three aroids from Bangladesh. Bangladesh J. Bot. 34: 115-120.

Deen, S. S. and Alam, Sk. S. 2002. Comparative study in two forms of Xanthosoma violaceum (Araceae) through karyotype and isozyme analysis. Bangladesh J. Bot. 31: 45-47.

Hizume, M., Abe, K. K. and Tanaka, A. 1988. Fluorescent chromosome bandings in the Taxodiaceae. La Kromosomo. 11-50: 1609-1619.

Huq, S., Ara, H., Hassan, A. and Alam, Sk. S. 2007. Comparative karyotype analysis of Typhonium trilobatum L. and its two morphological forms. Cytologia 72: 233-238.

Jessy, N. S., Begum, R., Khatun, M. and Alam, Sk. S. 2005. Differential fluoresent chromosome banding of four species in Haworthia Duval (Aloaceae). Cytologia 70: 435-440.

Kondo, T. and Hizume, M. 1982. Banding for the chromosomes of Cryptomeria japonica D. Don. J. J. For. Soc. 64: 356-358.

Schweizer, D. 1976. Reverse fluorescent chromosome banding with Chromomycin and DAPI. Chromosoma 58: $307-324$.

Sriboonma, D., Murata, J. and Iwatsuki, K. 1994. A revision of Typhonium (Araceae). J. Fac. Sci. Univ. Tokyo, Sect. 3: 255-313.

Stebbins, G. L. 1971. Chromosomal evolution in higher plants. Addison-Wesley publishing company, California. pp. 208.

Sumner, A. T. 1990. Chromosome Banding. Unwin Hyman Ltd. London. pp. 423. 